Nature manuscript 2016-01-00837z

\title{
An Autonomous Chemically Fuelled Small-Molecule Motor
}

\author{
Miriam R. Wilson ${ }^{1}$, Jordi Solà ${ }^{1,2 \ddagger}$, Armando Carlone ${ }^{1,3 \ddagger}$, Stephen M. Goldup ${ }^{1,4}$, Nathalie Lebrasseur ${ }^{1,5}$ \& David A. \\ Leigh $^{{ }^{*}}$ \\ ${ }^{1}$ School of Chemistry, University of Manchester, Oxford Road, Manchester, M13 9PL, UK \\ ${ }^{2}$ Current address: Institute of Advanced Chemistry of Catalonia (IQAC-CSIC), Jordi Girona 18-26, 08034 \\ Barcelona, Spain \\ ${ }^{3}$ Current address: Chirotech Technology Centre, Dr. Reddy's, Cambridge, CB4 OPE, UK \\ ${ }^{4}$ Current address: Department of Chemistry, University of Southampton, Southampton, SO17 1BJ, UK \\ ${ }^{5}$ Current address: School of Biological and Chemical Sciences, Queen Mary University of London, London, E1 \\ $4 N S, U K$ \\ $\$$ These authors contributed equally to the work.
}

Nature's molecular machines are amongst the most complex of all functional molecules and lie at the heart of virtually every biological process. ${ }^{1} \mathrm{~A}$ number of synthetic small-molecule machines have been developed previously, ${ }^{2}$ including molecular muscles, ${ }^{3,4}$ synthesizers,${ }^{5,6}$ pumps, $^{7-9}$ walkers, ${ }^{10}$ transporters $^{11}$ and light ${ }^{12-16}$ and electrically ${ }^{17}, 18$ driven rotary motors. However, although biological molecular motors are powered by chemical gradients or the hydrolysis of adenosine triphosphate (ATP), ${ }^{1}$ to date there are no synthetic smallmolecule motors that can operate autonomously using chemical energy (i.e. the components move directionally as long as a chemical fuel is present). ${ }^{19}$ Here we describe a system in which a small macrocycle is continuously transported directionally around a cyclic track when powered by irreversible reactions of a chemical fuel, fluorenylmethoxycarbonyl chloride (Fmoc-Cl). Key to the design is that the rate of reaction of Fmoc-Cl with reactive sites on the cyclic track is faster when the macrocycle is far from the reactive site than when it is close. We find that a bulky pyridine-based catalyst promotes carbonate-forming reactions that ratchet the displacement of the macrocycle away from the reactive sites on the track. Under reaction conditions where both attachment and cleavage of the Fmoc groups occur through different processes, and the cleavage reaction occurs at a rate independent of macrocycle location, net directional rotation of the molecular motor continues for as long as unreacted fuel remains. We anticipate that autonomous chemically-fuelled molecular motors will find application as engines for molecular nanotechnology., 19, 20

The design of nanoscale motors in which the components incessantly rotate with net directionality has tantalized scientists since Feynman's celebrated discussion of the physics of a theoretical tiny ratchet-and-pawl. ${ }^{21}$ In the 1990 s Kelly's group produced a series of molecular analogues of a ratchet-and-pawl, confirming the lack of directional bias 
in the movement of the components at equilibrium. ${ }^{22}$ Their designs culminated in a system that employed chemical reactions to bias a 120 degree rotation of a triptycene residue in one direction, ${ }^{23}$ but attempts to extend this approach to repetitive 360 degree directional rotation proved unsuccessful. ${ }^{24}$ Light-driven rotary molecular motors based on overcrowded alkenes ${ }^{12,13}$ and imines ${ }^{14,16}$ have been developed by the groups of Feringa and Lehn, while our group ${ }^{25,}$ ${ }^{26}$ and others ${ }^{27-29}$ have made molecules in which the components can be rotated directionally step-wise by repetitively carrying out several chemical reactions in sequence. The latter systems all operate through Brownian ratchet mechanisms, differentiating the rates of random thermal motion of the components in each direction by the manipulation of kinetic (mainly steric) barriers. ${ }^{20}$ Autonomous operation requires the ratchet mechanism to operate continuously, meaning that the barriers must be repeatedly raised and lowered under the same set of reaction conditions and coupled to the consumption of a chemical species in order to avoid falling foul of the Second Law of Thermodynamics. ${ }^{30}$

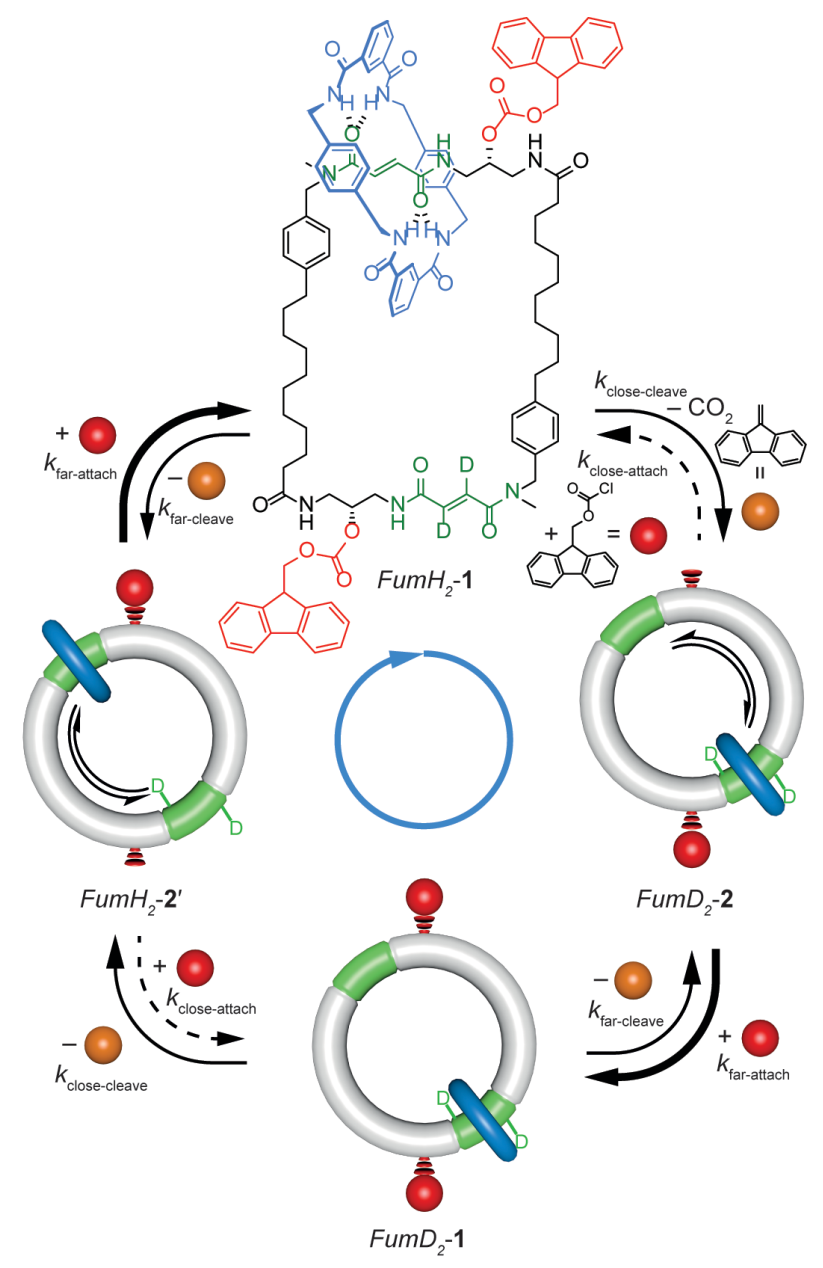

Figure 1 | Operation of a chemically-fuelled [2]catenane rotary motor. The benzylic amide macrocycle (blue) binds to one or other of the two fumaramide sites (green) of the cyclic track. Bulky groups (red) sterically block passage of the small blue ring and trap it in one compartment or the other (the right or left hand side of the track as shown). Cleavage of one of the bulky groups through a chemical reaction 
(loss of orange ball) allows the small ring to shuttle back-and-forth between the two fumaramide sites on the track via Brownian motion along the unblocked pathway. Attachment of another bulky group (addition of red ball) through another chemical reaction (under the same conditions) locks in any change of location of the small ring (i.e. if the ring has changed compartment it is prevented from returning to the original one). If the kinetics for blocking group attachment are faster when the small ring is far from the reactive site $\left(k_{\text {far-attach }}>k_{\text {close-attach }}\right.$; for example, for steric reasons), but the cleavage reaction occurs at a rate independent of the small ring position $\left(k_{\text {far-cleave }}=k_{\text {close-cleave }}\right)$, then the small ring will directionally rotate around the larger one. One of the fumaramide groups is deuterium labelled to distinguish the compartments and allow the location of the small ring to be determined by ${ }^{1} \mathrm{H}$ NMR spectroscopy. Compound $\mathbf{1}$ is the catenane with two Fmoc groups attached. Compound $\mathbf{2}$ is the catenane with one Fmoc group attached close to the labelled fumaramide group. Compound $\mathbf{2}$ ' is the catenane with one Fmoc group attached close to the unlabelled fumaramide group. The italicised prefix (FumH $2^{-}$or $\left.\mathrm{FumD}_{2^{-}}\right)$refers to the location of the benzylic amide macrocycle in $\mathbf{1}, \mathbf{2}$ or $\mathbf{2}^{\prime}$. Bold arrows indicate the major pathway of a reaction, dashed arrows the minor pathway and nonbolded arrows pathways that occur at similar rates. The blue arrow indicates the direction of net transport of the benzylic amide macrocycle when $k_{\text {far-attach }}>k_{\text {close-attach }}$ and $k_{\text {far-cleave }}=k_{\text {close-cleave }}$.

The structure and mechanism of operation of a rotary molecular motor (1) that continuously rotates its components with net directionality when driven by chemical energy is shown in Fig. 1. The molecule is a [2]catenane featuring two interlocked molecular rings of different sizes. Fumaramide residues (shown in green) on the larger ring (the 'track') serve as binding sites for a smaller benzylic amide macrocycle (blue). Removable bulky groups (red) block the passage of the small ring and, when both blocking groups are attached, trap it in one or other compartment of the cyclic track. As previously demonstrated, ${ }^{31,32}$ the macrocycle can be directionally transported between adjacent compartments of a rotaxane thread using the acylation of hydroxyl groups as the energy input. We reasoned that the issue of repeatedly raising and lowering the kinetic barriers to transport under a single set of reaction conditions could be achieved by using a blocking group that attaches and detaches through dissimilar reaction mechanisms: one reaction (e.g. attachment) proceeding at rates that vary according to the position of the small macrocycle, the other (e.g. cleavage) occurring at a rate independent of the small macrocycle position (an 'information ratchet' mechanism $^{7,20,31,32}$ ).

In [2] catenane $\mathbf{2} / \mathbf{2}^{\prime}$, in which one Fmoc group of $\mathbf{1}$ has been cleaved, there is a significant difference between the distances of the small-ring binding sites (fumaramide groups) and the revealed hydroxyl group; one is very close, where the presence of the ring should inhibit nucleophilic attack by the $\mathrm{OH}$ group on a large electrophile, and one too far away for a bound ring to significantly influence rates of reaction. This should result in dissimilar reaction rates for when the macrocycle occupies the fumaramide unit near $\left(k_{\text {close-attach }}\right)$ or far from $\left(k_{\text {far-attach }}\right)$ the hydroxyl group. We carried out model studies on a number of potential chemical fuels, eventually concentrating on 9fluorenylmethoxycarbonyl chloride $(\mathrm{Fmoc}-\mathrm{Cl})$ as its mechanism of attachment to the molecular motor is significantly different to that of cleavage of the resulting fluorenylmethoxycarbonate group (shown for the rotaxane model system 
in Fig. 2). The former occurs by nucleophilic attack of a hydroxyl group directly on the $\mathrm{C}=\mathrm{O}$ of the chloroformate residue, where the presence or absence of the bulky benzylic amide macrocycle on the adjacent fumaramide group would be expected to influence the reaction rate $\left(k_{\text {far-attach }} \neq k_{\text {close-attach }}\right)$. In contrast, the detachment reaction occurs by a reaction cascade (eliminating $\mathrm{CO}_{2}$ and dibenzofulvene) initiated by base abstraction of a proton from the fluorenyl methine group. This is five bonds remote from the site of attachment to the [2]catenane and so the influence of the position of the macrocycle on the detachment reaction rate should be minimal ( $\left.k_{\text {far-cleave }} \approx k_{\text {close-cleave }}\right)$. The reactions that lead to the attachment and to the cleavage of the Fmoc group can both be promoted under basic conditions.

Starting from the mono-hydroxyl species $\left(\mathbf{2}\right.$ and $\left.\mathbf{2}^{\prime}\right)$ Fmoc attachment to $\mathbf{2}$ should favour formation of FumD $\mathrm{D}_{2} \mathbf{- 1}$ (carbonate formation preferentially occurring distant to the small ring) and likewise Fmoc attachment to $\mathbf{2}^{\prime}$ should preferentially form $\mathrm{FumH}_{2} \mathbf{- 1}$, each reaction causing net transport of the benzylic amide macrocycle in a clockwise direction. The cleavage of either Fmoc group of 1 then occurs (to form $\mathbf{2}$ or $\mathbf{2}^{\prime}$ in equal amounts) setting the stage for another Fmoc attachment step to occur, again proceeding with net directional movement of the small ring. To maximize the efficiency of the process sufficient Fmoc-Cl needs to be present for the attachment reaction to proceed rapidly whenever a hydroxyl group is unmasked. This prevents accumulation of the catenane diol, in which both Fmoc groups have been cleaved, leaving the small ring free to shuttle around the track without directional bias. A fuller discussion of the kinetics ${ }^{33}$ of the information ratchet mechanism, including deriving the net directionality of ring rotation from the rate equations, is given in the Supplementary Information, Section S6.

We first developed the chemistry necessary for the operation of $\mathbf{1}$ on a simpler [2] rotaxane (a ring threaded on a dumbbell-shaped axle) system, 3 (Fig. 2). [2]Rotaxane 3 was prepared from (R)-3-amino-1,2-propanediol (see Supplementary Information, Section S1.2.1 and S1.3.1). When rotaxane 3 was treated with Fmoc-Cl in the presence of a bulky carbonate-forming catalyst, $(R)-5$ (Fig. 2a), the macrocycle was predominantly trapped in the FumD compartment (up to 17:83 FumH $_{2}-\mathbf{4}:$ FumD $_{2}-\mathbf{4}$, as evidenced by ${ }^{1} \mathrm{H}$ NMR, Fig. 2b. Other reaction conditions led to poorer discrimination between the compartments). This result confirms that catalyst $(R)-\mathbf{5}$, in its acylated intermediate form (Fig. 2a), can distinguish between the two positional isomers of the rotaxane that interconvert through the macrocycle shuttling between the two fumaramide residues, and preferentially reacts with the hydroxyl group when the macrocycle is on the $F u m D_{2}$ group, i.e. $k_{\text {far-attach }}>k_{\text {close-attach. Although a chiral catalyst (and chiral }}$ 
motor) was used, mainly for synthetic convenience, the positional bias of the Fmoc addition is almost independent of catalyst handedness (see Supplementary Information, Section S5) and stems from one macrocycle binding site being close to the site of reaction on the axle, the other far away.

With a directional bias established for the Fmoc addition step we next investigated the Fmoc cleavage reaction. A solution of 20:80 FumH ${ }_{2}-\mathbf{4}: F u m D_{2}-\mathbf{4}$, in dichloromethane $\left(\mathrm{CH}_{2} \mathrm{Cl}_{2}\right)$ was treated with triethylamine ( $\left.\mathrm{NEt}_{3}\right)(\mathrm{Fig}$. 2c). The reaction was sampled at various times and before all the rotaxane Fmoc groups had been cleaved ${ }^{1} \mathrm{H}$ NMR analysis of the recovered rotaxane 4 showed the ratio of $\mathrm{FumH}_{2}: \mathrm{FumD}_{2}$ to be unchanged from the starting ratio (e.g. rotaxane 4 after $67 \%$ formation of 3, Fig. 2d). Thus the Fmoc groups are cleaved from FumH $_{2}-\mathbf{4}$ and $\mathrm{FumD}_{2}-\mathbf{4}$ at the same rate; the position of the macrocycle in rotaxane 4 does not influence the rate of Fmoc cleavage, i.e. $k_{\text {far- }}$ cleave $=k_{\text {close-cleave }}$

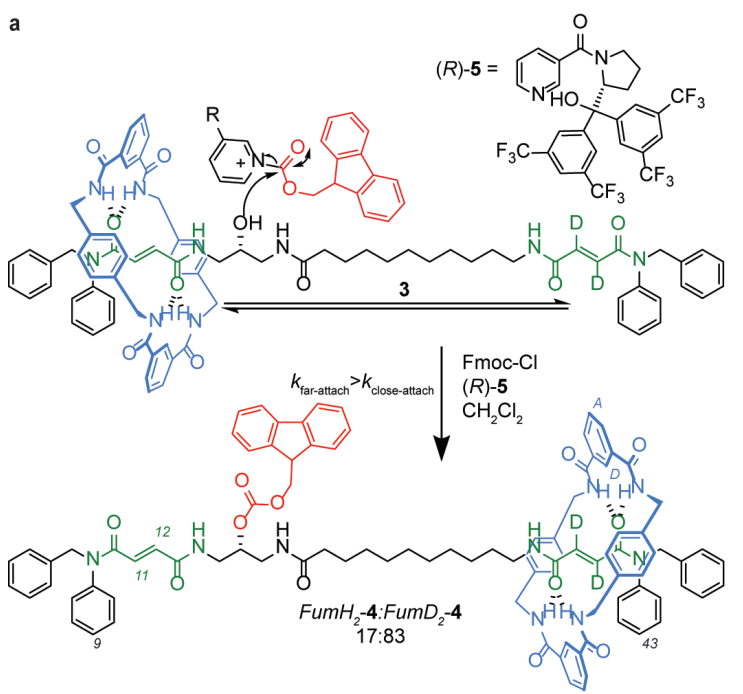
c

b i
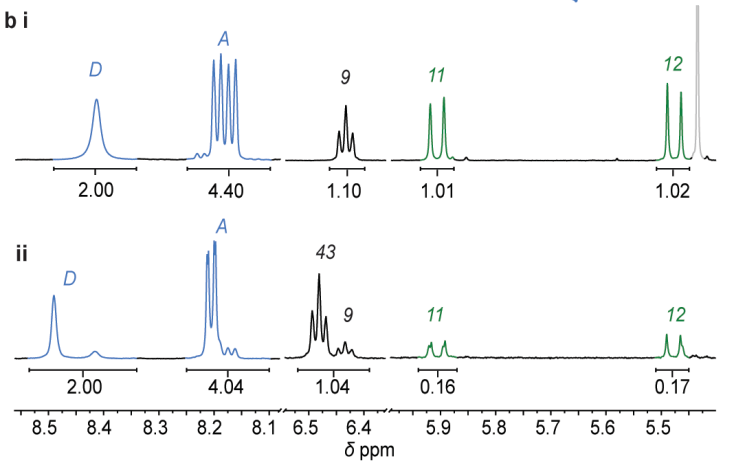

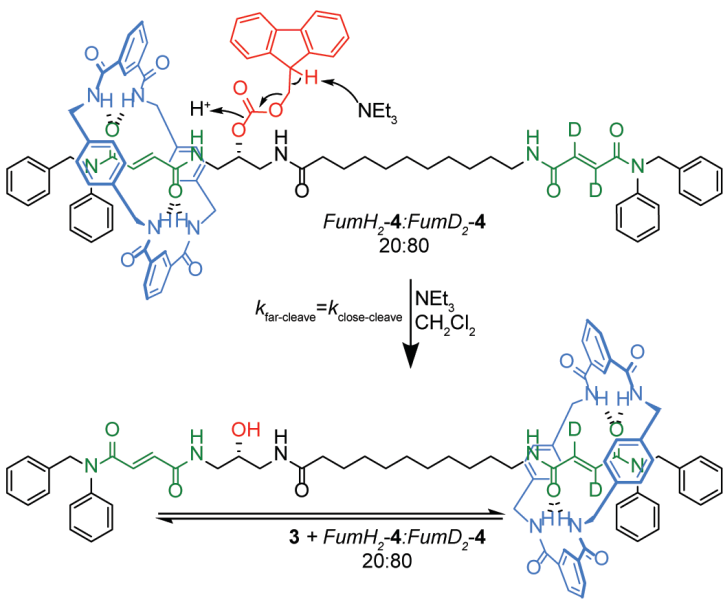

d i

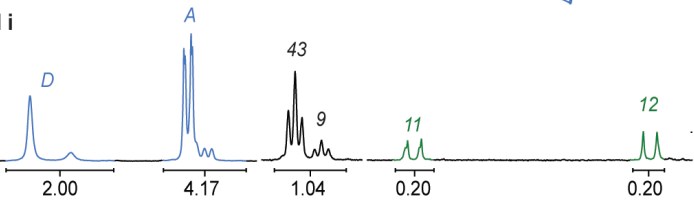

ii

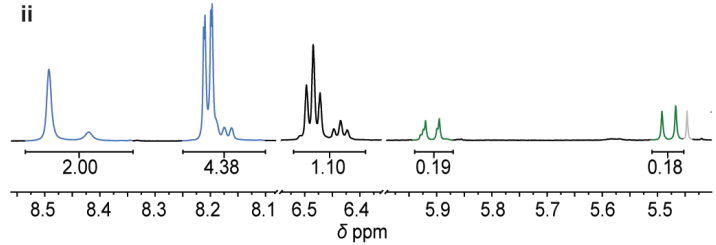

Figure 2 | [2]Rotaxane model system to demonstrate directional bias for Fmoc addition and position-independent Fmoc cleavage. a Positional bias of the macrocycle in Fmoc attachment to rotaxane 3. Reaction conditions: Fmoc-Cl (5 eq.), $(R)-5$ ( 5 eq. $), \mathrm{CH}_{2} \mathrm{Cl}$, r.t., 18 h. b Partial ${ }^{1} \mathrm{H}$ NMR spectra $\left(600 \mathrm{MHz}, \mathrm{CD}_{3} \mathrm{OD}: \mathrm{CDCl}_{3}\right.$ 3:1, $300 \mathrm{~K}$ ) of (i) 100:0 $\mathrm{FumH}_{2}-\mathbf{4}$ :FumD $\mathrm{F}_{2}-\mathbf{4}$ (obtained from an unambiguous synthetic route); (ii) 17:83 FumH $_{2}-\mathbf{4}: \mathrm{FumD}_{2}-\mathbf{4}$ formed using (R)-5 as the carbonate-forming catalyst. Residual solvent peaks are shown in grey. The lettering corresponds to the proton labelling in Figure 2a. Full spectral assignments are given in Supplementary Information, Section S1.3. In spectrum 2bii regions 6.3-6.6 and 5.4-6.0 ppm are scaled vertically $3 \times$ compared to region 8.0-8.6 ppm. c Lack of macrocycle positional bias for Fmoc cleavage from rotaxane 4. Reaction conditions: $\mathrm{NEt}_{3}\left(5\right.$ eq.), $\mathrm{CH}_{2} \mathrm{Cl}_{2}$, r.t., 2 h, 67 \%. d Partial ${ }^{1} \mathrm{H} \mathrm{NMR} \mathrm{spectra} \mathrm{(600} \mathrm{MHz}$, 
$\mathrm{CD}_{3} \mathrm{OD}: \mathrm{CDCl}_{3}$ 3:1, $300 \mathrm{~K}$ ) of (i) 20:80 FumH $\mathrm{Fum}_{2} \mathbf{- 4}: \mathrm{Fum}_{2}-\mathbf{4}$, (ii) $\mathrm{FumH}_{2}-\mathbf{4}: \mathrm{FumD}_{2}-\mathbf{4}$ recovered after cleavage of about two-thirds of the Fmoc groups. Residual solvent peaks are shown in grey. The lettering corresponds to the proton labelling in Fig. 2a. Regions 6.3-6.6 and 5.4-6.0 ppm are scaled vertically $3 \times$ compared to region $8.0-8.6 \mathrm{ppm}$.

Next, conditions were established under which both the Fmoc attachment and cleavage reactions take place in the same reaction mixture (see Supplementary Information, Section S2). In a typical procedure the rotaxane (3 or 4) and (R)-5 were dissolved in $\mathrm{CH}_{2} \mathrm{Cl}_{2}$ and $\mathrm{KHCO}_{3}$ added (to regenerate $\mathrm{NEt}_{3}$ from hydrochloride salts formed by the cleavage reaction). Solutions of the Fmoc-Cl fuel and $\mathrm{Et}_{3} \mathrm{~N}$ in $\mathrm{CH}_{2} \mathrm{Cl}_{2}$ were mixed together initially and then more Fmoc-Cl slowly and continuously added using a syringe pump for as long as the motor was required to run. Subjecting rotaxane 4 with an initial macrocycle distribution of 100:0 $\mathrm{FumH}_{2}: \mathrm{FumD}_{2}$ to these operation conditions resulted in 4 with a distribution of 17:83 FumH $\mathrm{FumD}_{2}$ at the steady state (Supplementary Figs. S3 \& S4). That the Fmoc formation and cleavage reactions run concurrently was further confirmed by showing that a deuterium-labelled Fmoc group on the rotaxane could be exchanged for an unlabelled one under these operating conditions. Treatment of $\mathrm{D}_{2}-\left(33: 67\right.$ FumH $_{2}:$ FumD $\left._{2}\right)-4$ with unlabelled Fmoc-Cl under the operating conditions formed (17:83 FumH $\left.\mathrm{F}_{2}: \mathrm{FumD}_{2}\right)-$ 4 with a loss of $\mathrm{D}_{2}$-label from $63 \%$ to $10 \%$ incorporation after $18 \mathrm{~h}$, as evidenced by mass spectrometry. Switching the chemical fuel being added to the deuterium-labelled version $\left(\mathrm{D}_{2}-\mathrm{Fmoc}-\mathrm{Cl}\right)$ then fully restored the labelled form of $\mathrm{D}_{2}-\left(17: 83\right.$ FumH $H_{2}:$ FumD $\left._{2}\right)-4$ after 66 h (see Supplementary Fig. S4).

We note that these results indicate that a macrocycle on a polymer consisting of repeat units of $\mathbf{4}$ without the terminal stopper groups should inexorably be transported towards one end of the polymer chain by treatment with the Fmoc$\mathrm{Cl}$ fuel under these reaction conditions. In other words, rotaxane $\mathbf{4}$ is a functioning engine system for a chemicallyfuelled linear molecular motor.

We applied the same principles to the synthesis and operation of a chemically-fuelled [2]catenane rotary molecular motor. [2]Catenane 2' was prepared from $(R)$-3-amino-1,2-propanediol (see Supplementary Information, Sections S1.2.2. and S1.3.2). The benzylic amide macrocycle distribution between the two fumaramide sites in $\mathbf{2}^{\prime}$ is approximately 40:60 FumH $\mathrm{FumD}_{2}$ (estimated from the ${ }^{1} \mathrm{H}$ NMR shielding of the $\mathrm{FumH}_{2}$ protons in 2' compared to $\mathrm{FumH}_{2}-\mathbf{1}$ and consistent with the results of carbonate formation promoted by pyridine, a small catalyst (Supplementary Table S2)). The energy barrier for macrocycle exchange between the fumaramide sites in related rotaxanes is $\sim 16 \mathrm{kcal} \mathrm{mol}^{-1}$ in $\mathrm{CDCl}_{3}{ }^{25}$ suggesting that macrocycle shuttling in $\mathbf{2}^{\prime}$ occurs hundreds of times a second under the motor operating conditions. 
The Fmoc attachment-cleavage chemistry of 2 (Supplementary Information, Section S3) mirrored that of rotaxane 3. When catenane $\mathbf{2}^{\prime}$ was treated with Fmoc-Cl in the presence of catalyst $(R)-\mathbf{5}$, the macrocycle was predominantly trapped in the $\mathrm{FumH}_{2}$ compartment (80:20 FumH $\mathrm{FumD}_{2} \mathbf{- 1} \mathbf{1}$ - $\mathbf{1}$, Supplementary Figs. S5a and b), i.e. $k_{\text {far-attach }}>k_{\text {close- }}$ attach. A model catenane was also prepared (see Supplementary Figs. S5c and d), replacing one Fmoc carbonate group of 1 with an analogous Fmoc-methinyl ester. The substitution of an oxygen atom for a carbon atom ensures that cleavage of this group cannot occur under the motor operating conditions. This enabled the Fmoc cleavage reaction to be studied in a catenane possessing only one detachable group. As with the rotaxane model, the control experiment demonstrated that the rate of Fmoc cleavage is not affected by the position of the macrocycle in the catenane (Supplementary Figs. 5 d), i.e. $k_{\text {far-cleave }}=k_{\text {close-cleave }}$.

To confirm that both the Fmoc addition and cleavage reactions take place with catenane 1, we first demonstrated that the reactions can occur sequentially (Fig. 3). Catenane 1 was treated with $\mathrm{NEt}_{3}$ (8 equiv.) and after $6 \mathrm{~h} 57 \%$ of the catenanes had lost one Fmoc group (forming 2/2'; Fig. 3, cycle 1) with a further $11 \%$ of catenanes having had both Fmoc groups cleaved. At this point deuterium-labelled fuel, $\mathrm{D}_{2}-\mathrm{Fmoc}-\mathrm{Cl}$, activated with $(R)-\mathbf{5}$ was added, leading to almost complete derivatisation of the catenane hydroxyl groups after $24 \mathrm{~h}(94 \% \mathrm{1})$. At the end of this cleavageaddition cycle (Fig. 3, cycle 1) electrospray mass spectrometry confirmed that the $\mathrm{D}_{2}$-labelled Fmoc groups had been incorporated from the fuel into the catenane motor (Fig. 3, cycle 1). The resulting mixture was then treated with a second cycle of $\mathrm{NEt}_{3}$, leading after $5 \mathrm{~h}$ to $55 \%$ of the catenane with only one Fmoc group, 2/2' (Fig. 3, cycle 2). Subsequent addition of unlabelled Fmoc-Cl regenerated $\mathbf{1}$ with a majority of the Fmoc groups without deuterium labels (Fig. 3, cycle 2). Thus over two complete operational cycles, the catenane molecules are shown to sequentially cleave and then add an Fmoc group from the fuel being supplied during that cycle, then cleave and add another Fmoc group from a second batch of fuel. 


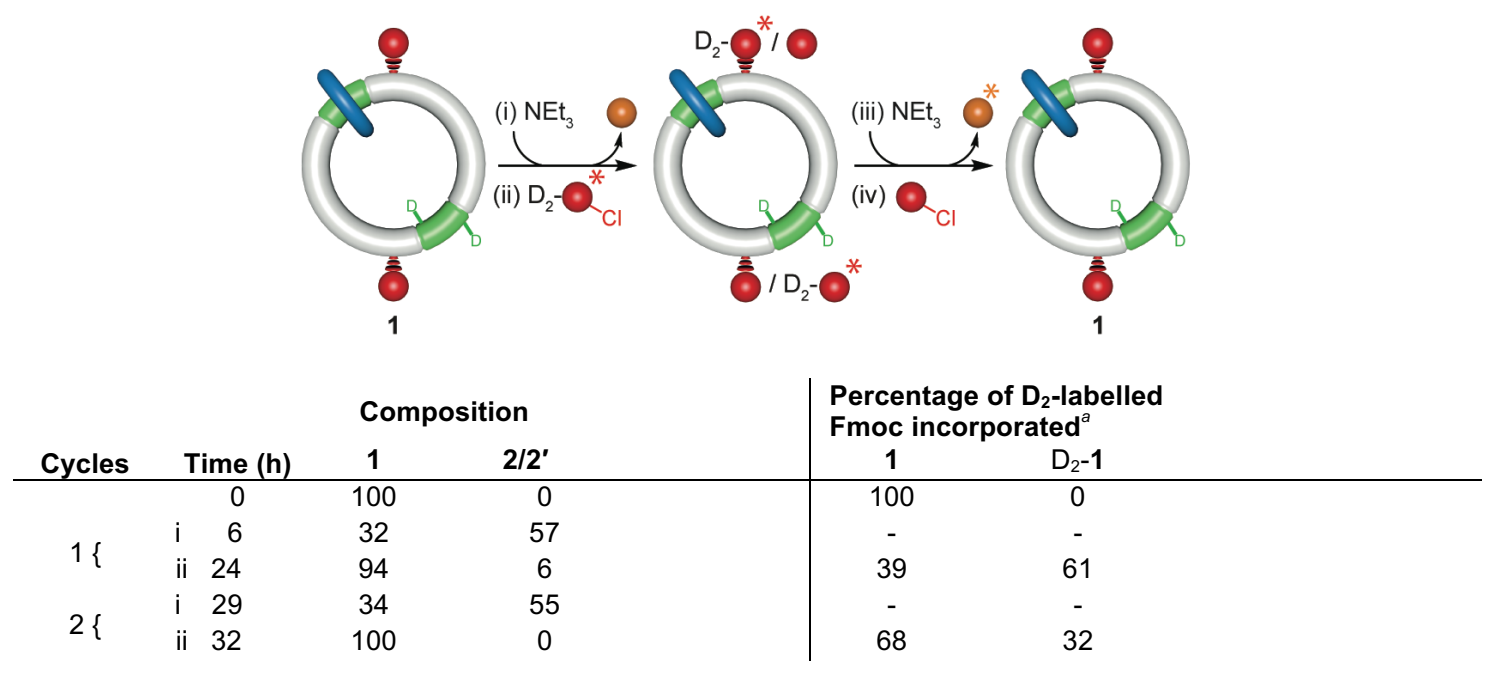

Figure 3 Exchange of Fmoc groups during stepwise operation of catenane 1. Reaction conditions: (i) $\mathrm{NEt}_{3}$ ( 8 eq.), (ii) $\mathrm{D}_{2}$-Fmoc-Cl (16 eq.), $(R)-5$ (16 eq.), (iii) $\mathrm{NEt}_{3}$ (10 eq.), (iv) Fmoc-Cl (16 eq.), $(R)-5$ (16 eq.). Diol (up to $11 \%$ ) is also formed during the Fmoc-cleavage steps under these conditions (10 eq. $\mathrm{NEt}_{3}$ ), see Supplementary Information, Table S1. ${ }^{a}$ Determined by relative intensity of the $[\mathrm{M}+\mathrm{Na}]^{+}$signal in electrospray mass spectrometry.

In order to monitor the catenane rotary motor during autonomous operation, catenane $\mathbf{1}$ with $80 \%$ of the small rings on the unlabelled fumaramide binding site $\left(80: 20 \mathrm{FumH}_{2}: \mathrm{FumD}_{2}-\mathbf{1}\right)$ was treated with $\mathrm{Fmoc}-\mathrm{Cl},(R)-\mathbf{5}, \mathrm{Et}_{3} \mathrm{~N}$ and $\mathrm{KHCO}_{3}$ in $\mathrm{CH}_{2} \mathrm{Cl}_{2}$ (Fig. 4). For autonomous operation we used conditions under which the Fmoc groups are added by the Fmoc-Cl fuel and cleaved with no discernible accumulation of diol (i.e. 1.5 equivalents $\mathrm{Et}_{3} \mathrm{~N}$ instead of the 8-10 equivalents employed in the sequential operations) and the distribution of the catenane positional isomers was measured over time by ${ }^{1} \mathrm{H}$ NMR spectroscopy (Fig. 4). Under these conditions the initial macrocycle distribution changed from 80:20 FumH $\mathrm{H}_{2}: \mathrm{FumD}_{2}$ to 55:45 (Fig. 4b). Shortly after the supply of Fmoc-Cl fuel is cut off, no further change in the distribution of the rings between the compartments occurs (i.e. the motor stops working). However, cleavage of the Fmoc groups slowly continues, unless the basic reaction medium is quenched, forming $\mathbf{2} / \mathbf{2}^{\prime}$ and eventually catenane diol.

The ratio of the distribution of the rings between the compartments falls towards $1: 1$ as a direct consequence of the functioning of the motor as each Fmoc-cleavage reaction serves to equilibrate the distribution of rings between the compartments. Although the Fmoc attachment reaction biases clockwise rotation of the small ring around the track, it does not bias its average position on the track. This is because Fmoc attachment to catenane 2' biases the small ring to the left hand compartment whereas Fmoc attachment to catenane 2 biases the small ring to the right hand compartment. As was demonstrated for rotaxane 4 (Fig. 2), the change in the macrocycle distribution that occurs with catenane 1 under the operating conditions (Fig. 4) shows that the Fmoc groups are being cleaved, allowing the small 
ring to move between compartments by Brownian motion, and the transiently generated hydroxyl groups are being derivatised under consumption of the $\mathrm{Fmoc}-\mathrm{Cl}$ fuel, i.e. confirming that the catenane rotary motor operates autonomously as long as unspent chemical fuel is present.
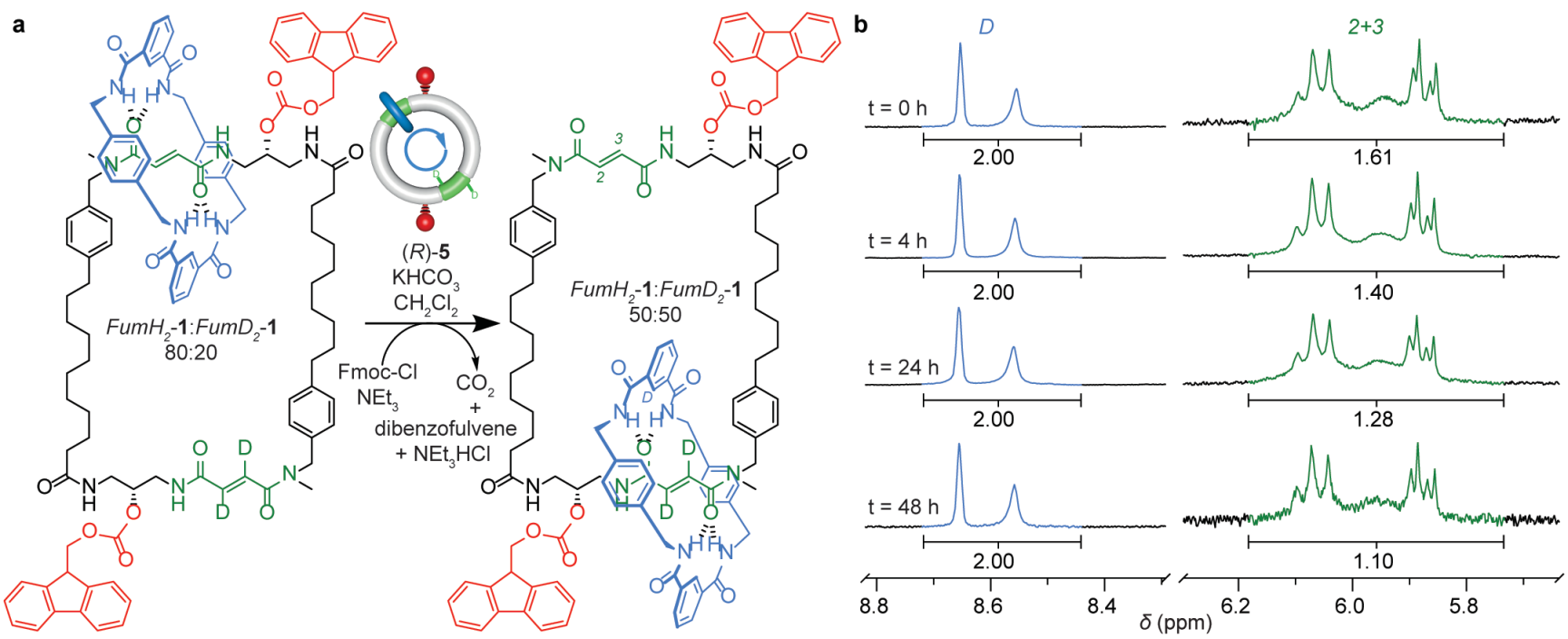

Figure 4 | Directional transport of the macrocycle monitored by ${ }^{1} \mathbf{H}$ NMR spectroscopy. a. Reaction conditions: (i) $(R)-5$ (5 eq.), $\mathrm{KHCO}_{3}$ (20 eq.), $\mathrm{CH}_{2} \mathrm{Cl}_{2}$, r.t., Fmoc-Cl, $\mathrm{CH}_{2} \mathrm{Cl}_{2}$, added via syringe pump at 2.4 eq./h, then $\mathrm{NEt}_{3}$ (1.5 eq.) after 1 hour of Fmoc-Cl addition. b. Partial ${ }^{1} \mathrm{H}$ NMR spectra $\left(500 \mathrm{MHz}, \mathrm{CD}_{2} \mathrm{Cl}_{2}: \mathrm{CD}_{3} \mathrm{OD}: 1: 1,300 \mathrm{~K}\right)$ of 80:20 Fum $\mathrm{H}_{2}-\mathbf{1}$ :FumD ${ }_{2}-1$ and after operation for $4 \mathrm{~h}, 24 \mathrm{~h}$ and $48 \mathrm{~h}$. The region 5.7$6.3 \mathrm{ppm}$ is scaled vertically $6 \times$ compared to region $8.3-8.8 \mathrm{ppm}$. The two macrocycle positional isomers of catenane 1 each exist as four tertiary amide rotamers.

Proving directional rotation in molecular motors is challenging, not least because each rotational cycle returns the motor components to their starting positions. Evidence for directionality in step-wise operated small-molecule motors has previously been provided by determining the position of the components at multiple points in a motor's cycle and determining the rates of different pathways to those positions. ${ }^{12,25}$ However, in a continuously operating motor with only two minimum energy positions of the components, such as $\mathbf{1}$, this approach is not possible. Nevertheless, fueldriven directional rotation in $\mathbf{1}$ could be unequivocally established through a series of individually provable premises, a form of deductive logic commonly used in mathematical proofs. If all of the premises are experimentally demonstrated to be correct, and the terms linking the premises to the conclusion are valid, then the conclusion reached is necessarily true. Net directional rotation of the chemically-fuelled rotary motor $\mathbf{1}$ through an information ratchet mechanism (see Supplementary Information, Section S6, for an explanation of how directionality intrinsically follows from the rate equations) is demonstrated through experimental verification of each of three premises: 
(1) Under the motor operating conditions the Fmoc attachment reaction to the catenane (from Fmoc-Cl) and the Fmoc cleavage reaction from the catenane (forming $\mathrm{CO}_{2}$ and dibenzofulvene) both occur.

This is shown by the experiments that demonstrate that $\mathrm{D}_{2}$-Fmoc groups add to the catenane when using $\mathrm{D}_{2}$-Fmoc fuel and are then replaced by unlabelled Fmoc groups upon switching to unlabelled fuel (Fig. 3). Fmoc-Cl is not simply being destroyed in the reaction, it is being continuously added and cleaved from the catenane under the operating conditions.

(2) Under the motor operating conditions Fmoc attachment to the catenane hydroxyl group (2 or $\left.\mathbf{2}^{\prime}\right)$ results in a bias in the distribution of the macrocycle between the compartments in the resulting di-Fmoc catenane (1) (i.e. $k_{\text {far- }}$

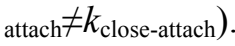

This is shown by the positional bias in the Fmoc attachment to $\mathbf{2}^{\prime}$ experiments (Supplementary Figs. 5a and b), analogous to that shown for rotaxane 3 in Fig. 2a.

(3) Under the motor operating conditions cleavage of one Fmoc group from catenane $\mathbf{1}$ occurs at a rate independent of the position of the macrocycle (i.e. $k_{\text {far-cleave }}=k_{\text {close-cleave }}$ ).

This is proven by the catenane Fmoc cleavage experiments (Supplementary Figs. 5c and d), analogous to that shown for rotaxane 20:80 FumH $\mathrm{F}_{2}: \mathrm{FumD}_{2}-4$ in Fig. 2c.

The effects of the net-directional movement of the rings around the catenane track are directly observed in the experiment shown in Fig. 4. The catenane ring distribution can only change through the benzylic amide macrocycles shuttling between the fumaramide sites when an Fmoc group is transiently cleaved, and the directional bias of the ring movement under these conditions is that experimentally determined in proving premises (2) and (3).

Chirality is not necessary for directional rotation: the wheels of a bicycle travelling down a road rotate clockwise with respect to an observer of one side of the road and counter-clockwise with respect to an observer on the other. ${ }^{25}$ However, the chiral centres of $\mathbf{1}$ differentiate the two faces of the track, defining the direction of the ring rotation in $\mathbf{1}$ as clockwise with respect to the $(R, R)$-stereochemistry of the molecular motor. 
Just as motor proteins are catalysts for the hydrolysis of ATP, the catenane (1) and rotaxane (4) motors are catalysts for the conversion of Fmoc- $\mathrm{Cl}$ and $\mathrm{Et}_{3} \mathrm{~N}$ into dibenzofulvalene, $\mathrm{CO}_{2}$ and $\mathrm{Et}_{3} \mathrm{NHCl}$. For both the biological and synthetic motors it is the free energy released by the motor-catalysed exergonic reactions that drives the directional displacement of the motor components. In principle two molecules of $\mathrm{Fmoc}-\mathrm{Cl}$ are required to power one 360 degree clockwise ratcheted rotation of the benzylic amide macrocycle around the catenane track of $\mathbf{1}$. In practice the directionality of the fuelled rotation is good: the 80:20 positional bias observed in the Fmoc-attachment reaction means that for every ten molecules of Fmoc-Cl that react with the track the benzylic amide macrocycle makes on average three net directional full rotations about the track. However, unlike motor proteins, ${ }^{20}$ rotaxane $\mathbf{3}$ and catenane 2/2' are poor catalysts for the destruction of their chemical fuel, and $\mathbf{1}$ and $\mathbf{4}$ only react with base to form $\mathrm{CO}_{2}$ and dibenzofulvene a few times faster than the background base-promoted decomposition of Fmoc-Cl.

From the rate at which the ratio of the macrocycle distribution between the compartments in the catenane falls to unity, the speed of net-directional rotation in the experiment shown in Fig $4 \mathrm{~b}$ can be calculated to be $\sim 12$ hours for each 360 degree rotation. This might be increased by raising the temperature and/or increasing the concentration and/or rate of addition of the fuel, but changes in these parameters might also affect the net-directionality of rotation. An investigation of these factors is ongoing in our laboratory.

Synthetic chemically fuelled molecular motors $\mathbf{1}$ and $\mathbf{4}$ join light-driven molecular rotary motors as engines with the potential to power tasks in molecular nanotechnology. ${ }^{19}$ Finding ways to link the position of the ring to more effective catalytic decomposition of the fuel should allow for the development of faster and more efficient smallmolecule motors powered by chemical fuels.

\section{Methods}

General method for autonomous operation of rotary catenane motor 1 . To a solution of 1 ( $5 \mathrm{mg}, 2.6 \mu \mathrm{mol})$ in $\mathrm{CH}_{2} \mathrm{Cl}_{2}(0.3 \mathrm{~mL})$ was added $(R)-5(5$ eq., $8.2 \mathrm{mg}, 13.0 \mu \mathrm{mol})$ and $\mathrm{KHCO}_{3}(20$ eq., $5.2 \mathrm{mg}, 52 \mu \mathrm{mol})$. A solution of Fmoc-Cl $(240 \mathrm{mg}, 0.93 \mathrm{mmol})$ in $\mathrm{CH}_{2} \mathrm{Cl}_{2}(1.0 \mathrm{~mL})$ was added at a rate of $6.7 \mu \mathrm{L} / \mathrm{h}$. After 1 hour $\mathrm{NEt}_{3}(1.5$ eq., 0.55 $\mu \mathrm{L}, 3.9 \mu \mathrm{mol})$ was added and Fmoc-Cl addition continued at a rate of $6.7 \mu \mathrm{L} / \mathrm{h}(6.2 \mu \mathrm{mol} / \mathrm{h}$ Fmoc-Cl for $2.6 \mu$ mol 1) for as long as the motor was required to run. After full consumption of the chemical fuel (Fmoc-Cl) the catenane motor was recovered by addition of $1 \mathrm{M} \mathrm{HCl}$ (aq.) $(10 \mathrm{ml})$ and the aqueous layer extracted with $\mathrm{CH}_{2} \mathrm{Cl}_{2}(3 \times 20 \mathrm{ml})$. 
The combined organic layers were washed with brine, dried over $\mathrm{Na}_{2} \mathrm{SO}_{4}$ and concentrated under reduced pressure. Purification by preparative TLC $\left(\mathrm{SiO}_{2}, \mathrm{CH}_{2} \mathrm{Cl}_{2}: \mathrm{EtOH} 95: 5\right)$ gave pristine 1.

\section{References}

1. Schliwa, M. \& Woehlke, G. Molecular motors. Nature 422, 759-765 (2003).

2. Erbas-Cakmak, S., Leigh, D. A., McTernan, C. T. \& Nussbaumer, A. L. Artificial molecular machines. Chem. Rev. 115, 10081-10206 (2015).

3. Jiménez, M. C., Dietrich-Buchecker, C. \& Sauvage, J.-P. Towards synthetic molecular muscles: contraction and stretching of a linear rotaxane dimer. Angew. Chem. Int. Ed. 39, 3284-3287 (2000).

4. Bruns, C. J. \& Stoddart, J. F. Rotaxane-based molecular muscles. Acc. Chem. Res. 47, 2186-2199 (2014).

5. Thordarson, P., Bijsterveld, E. J. A., Rowan, A. E. \& Nolte, R. J. M. Epoxidation of polybutadiene by a topologically linked catalyst. Nature 424, 915-918 (2003).

6. Lewandowski, B. et al. Sequence-specific peptide synthesis by an artificial small-molecule machine. Science 339, 189-193 (2013).

7. Serreli, V., Lee, C.-F., Kay, E. R. \& Leigh, D. A. A molecular information ratchet. Nature 445, 523-527 (2007).

8. Ragazzon, G., Baroncini, M., Silvi, S., Venturi, M. \& Credi, A. Light-powered autonomous and directional molecular motion of a dissipative self-assembling system. Nature Nanotech. 10, 70-75 (2015).

9. Cheng, C. et al. An artificial molecular pump. Nature Nanotech. 10, 547-553 (2015).

10. von Delius, M., Geertsema, E. M. \& Leigh, D. A. A synthetic small molecule that can walk down a track. Nature Chem. 2, 96-101 (2010).

11. Kassem, S., Lee, A. T. L., Leigh, D. A., Markevicius, A. \& Solà, J. Pick-up, transport and release of a molecular cargo using a small-molecule robotic arm. Nature Chem. 8, 138-143 (2016).

12. Koumura, N., Zijlstra, R. W. J., van Delden, R. A., Harada, N. \& Feringa, B. L. Light-driven monodirectional molecular rotor. Nature 401, 152-155 (1999).

13. Eelkema, R. et al. Molecular machines: Nanomotor rotates microscale objects. Nature 440, 163 (2006).

14. Greb, L. \& Lehn, J.-M. Light-driven molecular motors: Imines as four-step or two-step unidirectional rotors. $J$. Am. Chem. Soc. 136, 13114-13117 (2014).

15. Li, Q., et al. Macroscopic contraction of a gel induced by the integrated motion of light-driven molecular motors. 
Nature Nanotech. 10, 161-165 (2015).

16. Greb, L., Eichhöfer, A. \& Lehn, J.-M. Synthetic molecular motors: Thermal N inversion and directional photoinduced $\mathrm{C}=\mathrm{N}$ bond rotation of camphorquinone imines. Angew. Chem. Int. Ed. 54, 14345-14348 (2015).

17. Tierney, H. L. et al. Experimental demonstration of a single-molecule electric motor. Nature Nanotech. 6, 625$629(2011)$.

18. Perera, U. G. E., Ample, F., Kersell, H., Zhang, Y., Vives, G., Echeverria, J., Grisolia, M., Rapenne, G., Joachim, C. \& Hla, S.-W. Controlled clockwise and anticlockwise rotational switching of a molecular motor. Nature Nanotech. 8, 46-51 (2013).

10. Kay, E. R \& Leigh, D. A. Rise of the molecular machines. Angew. Chem. Int. Ed. 54, 10080-10088 (2015).

20. Astumian, R. D. Microscopic reversibility as the organizing principle of molecular machines. Nature Nanotechnol. 7, 684-688 (2012).

21. Feynman, R. P., Leighton, R. B. \& Sands, M. The Feynman Lectures on Physics (Addison-Wesley, Reading, MA, 1963), vol. 1, chap. 46.

22. Kelly, T. R., Tellitu, I. \& Sestelo, J. P. In search of molecular ratchets. Angew. Chem., Int. Ed. Engl. 36, 18661868 (1997).

23. Kelly, T. R., De Silva, H. \& Silva, R. A. Unidirectional rotary motion in a molecular system. Nature 401, 150152 (1999).

24. Kelly, T. R., et al. Progress toward a rationally designed, chemically powered rotary molecular motor. J. Am. Chem. Soc. 129, 376-386 (2007).

25. Leigh, D. A., Wong, J. K. Y., Dehez, F. \& Zerbetto, F. Unidirectional rotation in a mechanically interlocked molecular rotor. Nature 424, 174-179 (2003).

26. Hernández, J. V., Kay, E. R. \& Leigh, D. A. A reversible synthetic rotary molecular motor. Science 306, 15321537 (2004).

27. Fletcher, S. P., Dumur, F., Pollard, M. M. \& Feringa, B. L. A reversible, unidirectional molecular rotary motor driven by chemical energy. Science 310, 80-82 (2005).

28. Haberhauer, G. A molecular four-stroke motor. Angew. Chem. Int. Ed. 50, 6415-6418 (2011).

29. Lu, C.-H., Cecconello, A., Elbaz, J., Credi, A. \& Willner, I. A three-station DNA catenane rotary motor with controlled directionality. Nano Lett. 13, 2303-2308 (2013). 
30. Davis, A. P. Tilting at windmills? The second law survives. Angew. Chem. Int. Ed. 37, 909-910 (1998).

31. Alvarez-Pérez, M., Goldup, S. M., Leigh, D. A. \& Slawin, A. M. Z. A chemically-driven molecular information ratchet. J. Am. Chem. Soc. 130, 1836-1838 (2008).

32. Carlone, A., Goldup, S. M., Lebrasseur, N., Leigh, D. A. \& Wilson, A. A three-compartment chemically-driven molecular information ratchet. J. Am. Chem. Soc. 134, 8321-8323 (2012).

33. Cheng, C., McGonigal, P. R., Stoddart, J. F. \& Astumian, R. D. Design and synthesis of nonequilibrium systems. ACS Nano 9, 8672-8688 (2015).

\section{Supplementary Information}

Detailed synthetic procedures, operation methods, full characterisation data, ratchet mechanism kinetics.

\section{Author Contributions}

MRW, AC, JS, SMG and NL carried out the experimental work. MRW and JS designed and performed the operation experiments. DAL directed the research. All the authors contributed to the analysis of the results and the writing of the manuscript.

\section{Corresponding Author}

david.leigh@manchester.ac.uk.

\section{Notes}

The authors declare no competing financial interests.

\section{Acknowledgements}

We thank Dean R. Astumian for the analysis of the catenane motor reaction kinetics, the European Research Council (ERC) for funding and the EPSRC National Mass Spectrometry Service Centre (Swansea, UK) for high resolution mass spectrometry. 